\title{
Local Disaggregation of Negative Demand and Excess Demand Functions
}

Chiappori, Pierre-André; Ekeland, Ivar; Browning, Martin

Publication date:

2005

Document version

Publisher's PDF, also known as Version of record

Citation for published version (APA):

Chiappori, P-A., Ekeland, I., \& Browning, M. (2005). Local Disaggregation of Negative Demand and Excess Demand Functions. (2005-09 ed.) Department of Economics, University of Copenhagen. 


\section{CAM}

\section{CAM}

Centre for Applied

Microeconometrics

Institute of Economics

University of Copenhagen

http://www.econ.ku.dk/CAMI

Local Disaggregation of Negative Demand and Excess Demand Functions

Pierre-André Chiappori, Ivar Ekeland and Martin Browning

2005-09 


\title{
Local disaggregation of negative demand and excess demand functions
}

\author{
Pierre-Andre Chiappori $^{*} \quad$ Ivar Ekeland $^{\dagger} \quad$ Martin Browning $^{\ddagger}$
}

May 2005

\begin{abstract}
The literature on the characterization of aggregate excess and market demand has generated three types of results: global, local, or 'at a point'. In this note, we study the relationship between the last two approaches. We prove that within the class of functions satisfying standard conditions and whose Jacobian matrix is negative semi-definite, only $n / 2+1$ agents are needed for the 'at' decomposition. We ask whether, within the same class, the 'around' decomposition also requires only $n / 2+1$ agents.

Keywords: aggregation; market demand functions.

JEL classification: D510
\end{abstract}

\footnotetext{
*Department of Economics, Columbia University. Email: pc2167@columbia.edu

$\dagger$ University of British Columbia. Email: ekeland@math.ubc.ca

$\ddagger$ Institute of Economics, University of Copenhagen, Studiestraede 6, DK-1455 Copenhagen K, Denmark
} 


\section{Introduction}

The literature on the characterization of aggregate excess and market demand has generated three types of results: global, local, or 'at a point'. Global results provide necessary and sufficient conditions for a given function to coincide with the aggregate excess (respectively market) demand of a $n$-commodity economy within some given, possibly 'large' domain of prices. Debreu (1974) and Mantel (1974) show, for instance, that any continuous and homogeneous mapping of $\mathbb{R}^{n}$ that satisfies Walras Law can be decomposed as the aggregate excess demand of an economy with $k \geq n$ agents on any open subset of the positive orthant. No result of this kind exists for market demand, essentially because of non negativity constraints; indeed, Sonnenschein, in his initial contribution (1973a), provides an example of mapping that cannot be globally decomposed as the market demand of an economy, whatever the number of agents. Local results provide conditions for the existence of a decomposition of this type within some neighbourhood of a given point. For instance, Geanakoplos and Polemarchakis (1980) have proved that $n-1$ agents are sufficient for solving the excess demand problem in the neighbourhood of any price vector where aggregate demand is not zero. Similarly, Chiappori and Ekeland (1999) have recently demonstrated that any analytic mapping of $\mathbb{R}^{n}$ that satisfies adding up can be decomposed as the market demand of some $n$-consumer economy in the neighbourhood of any price vector.

Finally, several authors (Sonnenschein 1974, Mantel 1977, Diewert 1977, Geanakoplos and Polemarchakis 1980) have derived conditions under which, at some given point, an arbitrary mapping behaves 'as if' it was the aggregate (excess or market) demand of an economy, in the sense that both the value of the function and its (first) derivatives coincide with those of an aggregate demand. This is sometimes referred to as the 'at a point' approach (as opposed to the local, 'around a point' one).

However, the link between the last two types of results ('around a point' versus 'at a point') has not been fully understood yet. An open question is whether the local decomposition theorems ('around') are simply some non linear extension of the 'point wise' ('at') approach, in the sense that the same conditions that were necessary and sufficient in the latter case turn out to be also necessary and sufficient for the former problem. In other words, take a function such that the 'at' problem has a solution at any point in a neighbourhood of some given $\bar{p}$. Is it always possible to extend (one of) the solutions to the 'at' problem at $\bar{p}$, in order to get a solution to the 'around' problem? 
The goal of this note is to contribute to this issue by proving a result and asking a question. The result can be stated as follows. Take some arbitrary price vector $\bar{p}$ and some arbitrary function $X(p)$ satisfying the standard conditions (differentiability or smoothness and adding-up, plus homogeneity in the excess demand case). Assume, in addition, that $D_{p} X(\bar{p})$ is negative semidefinite. Then, for any $k \geq n / 2+1$, it is always possible to find an economy with $k$ agents such that the value of the aggregate demand and of its first derivatives coincide with that of $X(p)$. In other words, within the class of function satisfying standard conditions and with a negative Jacobian matrix at $\bar{p}$, only $n / 2+1$ agents (at most) are needed for the 'at' approach. The question is whether the same result holds for local decomposition. Within the class of functions satisfying standard conditions and the Jacobian matrix of which is negative definite at $\bar{p}$, is it the case that only $k=n / 2+1$ agents (at most) are needed to decompose the function, in some arbitrarily small neighbourhood of $\bar{p}$, as the aggregate market demand of an economy with $k$ agents?

\section{The basic framework}

In what follows, all results are derived in the case of market demand; the extension to excess demand is straightforward and left to the reader.

\subsection{The problem}

Take some continuously differentiable mapping $\mathbf{X}(\mathbf{p}): \mathbb{R}^{n} \rightarrow \mathbb{R}^{n}$ satisfying addingup:

$$
\text { p. } \mathbf{X}(\mathbf{p})=1
$$

and choose some arbitrary price vector $\overline{\mathbf{p}}$ in $\mathbb{R}_{+}^{n}$.

The 'around' problem can be stated as follows : under which conditions is it possible to find an open neighbourhood $\mathcal{V}(\overline{\mathbf{p}})$ and $k$ individual demand functions $\mathbf{x}^{1}(\mathbf{p}), \ldots, \mathbf{x}^{k}(\mathbf{p})$ such that, for any $\mathbf{p}$ within $\mathcal{V}(\overline{\mathbf{p}})$

$$
\mathbf{X}(\mathbf{p})=\mathbf{x}^{1}(\mathbf{p})+\ldots+\mathbf{x}^{k}(\mathbf{p})
$$

The 'at' problem can be stated as follows : under which conditions is it possible to find $k$ individual demand functions $\mathbf{x}^{1}(\mathbf{p}), \ldots, \mathbf{x}^{k}(\mathbf{p})$ such that

$$
\mathbf{X}(\overline{\mathbf{p}})=\mathbf{x}^{1}(\overline{\mathbf{p}})+\ldots+\mathbf{x}^{k}(\overline{\mathbf{p}})
$$




$$
D_{p} \mathbf{X}(\overline{\mathbf{p}})=\stackrel{\text { and }}{=} D_{p} \mathbf{x}^{1}(\overline{\mathbf{p}})+\ldots+D_{p} \mathbf{x}^{k}(\overline{\mathbf{p}})
$$

In both cases, $\mathbf{x}^{i}(\mathbf{p})$ must be a demand function, i.e., must solve

$$
\begin{array}{r}
V^{i}(\mathbf{p})=\max U^{i}\left(\mathbf{x}^{i}\right) \\
\mathbf{p} \cdot \mathbf{x}^{i}=\frac{1}{k} \\
\mathbf{x}^{i} \geq 0
\end{array}
$$

for some well-behaved utility function $U^{i}$.

Obviously, any solution to the 'around' problem provides a solution to the 'at' problem. However, whether, conversely, the existence of solutions to the 'at'

problem is sufficient for the existence of a solution to the 'around' problem is not known.

\subsection{Decomposition 'at' $\overline{\mathrm{p}}$ : the basic result}

We first state the basic result of the 'at' approach. Assume that the 'at' problem has a solution, and that each corresponding indirect utility $V^{i}$ is convex and twice continuously differentiable. From the envelope theorem, (5) implies that:

$$
D_{p} V^{i}(p)=-\lambda_{i}(p) x^{i}(p)
$$

where $D_{p} V$ denotes the gradient of $V$ and where the scalar $\lambda_{i}(p)$ is the Lagrange multiplier associated with the program. This, in turn, leads to (with obvious notations):

$$
D_{p}^{2} V^{i}(p)=-\lambda_{i}(p) D_{p} x^{i}(p)-x^{i}(p) D_{p} \lambda_{i}(p)^{\prime}
$$

or

$$
D_{p} x^{i}(p)=\frac{1}{\lambda_{i}(p)}\left[-D_{p}^{2} V^{i}(p)-x^{i}(p) D_{p} \lambda_{i}(p)^{\prime}\right]
$$

Then (4) becomes:

$$
D=D_{p} \mathbf{X}(\overline{\mathbf{p}})=\sum_{i=1}^{k} \frac{-1}{\lambda_{i}(\overline{\mathbf{p}})} D_{p}^{2} V^{i}(\overline{\mathbf{p}})-\sum_{i=1}^{k} \frac{1}{\lambda_{i}(\overline{\mathbf{p}})} x^{i}(\overline{\mathbf{p}}) D_{p} \lambda_{i}(\overline{\mathbf{p}})^{\prime}
$$

Consider the right-hand side of this equation. The first sum is a symmetric, negative semidefinite matrix, whereas the second sum is a matrix of rank at most $k$. In particular, let

$$
E=x^{1}(\overline{\mathbf{p}})^{\perp} \cap \ldots \cap x^{k}(\overline{\mathbf{p}})^{\perp}
$$


denote the set of vectors orthogonal to all individual demands; note that the dimension of $E$ is at least $n-k$. Then the restriction to $E$ of the matrix $D=$ $D_{p} \mathbf{X}(\overline{\mathbf{p}})$ is symmetric and negative semidefinite, i.e.,

$$
\begin{aligned}
\forall v, w & \in E, \quad v^{\prime} D w=w^{\prime} D v \\
\forall v & \in E, \quad v^{\prime} D v \leq 0
\end{aligned}
$$

When individual demand are not observable, one can state the following result:

Proposition 2.1. (Diewert 1977) Assume that some matrix $D$ is the Jacobian matrix, at some point $\overline{\mathbf{p}}$, of the aggregate demand function $X(p)$ of an economy with $k$ agents. Then there must exist some subspace $E$, of codimension at most $k$, that is included in $X(p)^{\perp}$ and such that the restriction to $E$ of $D$ is symmetric and negative semidefinite.

While this condition is obviously necessary for both the 'at' and the 'around' approaches, it turns out to be also sufficient for the 'at' result, as proved by Mantel (1977) for market demand and Geanakoplos and Polemarchakis (1980) for excess demand.

\section{Symmetry: how many agents?}

Proposition 2.1 requires two properties, symmetry and negativeness, that appear to play very different roles. Clearly, one cannot guarantee negativeness unless $k=n$. Indeed, choose a function $X(p)$ such that $D=D_{p} \mathbf{X}(\overline{\mathbf{p}})$ is positive definite. Then the conditions of Proposition 2.1 cannot be fulfilled unless $E$ is of dimension zero. An illustration, in the excess demand context, is provided by Debreu's celebrated example of a function that cannot be globally decomposed as the excess demand function of an economy with $k$ agents if $k<n$. It can readily be checked that function he considers has a positive definite Jacobian on the orthogonal of the price vector, and that this property is crucial for the argument ${ }^{1}$.

The case of symmetry is more complex and more surprising, since only $(n+1) / 2$ agents (and sometimes only $n / 2$ ) are needed. The precise results are the following:

Proposition 3.1. For any $(n \times n)$ matrix $D$, where $n$ is even, one can find a space $F$ of dimension $k \geq n / 2$ such that the restriction to $F$ of the mapping $D$

\footnotetext{
${ }^{1}$ In Debreu's proof, the contradiction obtains from a violation of the weak axiom of revealed preferences, that is equivalent to negativeness of the Slutsky matrix.
} 
is symmetric. For any $(n \times n)$ matrix $D$, where $n$ is odd, one can find a space $F$ of dimension $k \geq(n+1) / 2$ such that the restriction to $F$ of the mapping $D$ is symmetric.

Proof. See appendix

An immediate corollary is the following:

Corollary 3.2. Let $\overline{\mathbf{p}}$ be any point in $\mathbb{R}_{+}^{n}$ and let $X(p)$ be some $C^{1}$ function satisfying adding-up and such that $D=D_{p} \mathbf{X}(\overline{\mathbf{p}})$ is negative semi-definite. Then:

- if $n$ is even, then for any $k \geq n / 2+1$, it is possible to find an economy with $k$ consumers that solves the 'at $\overline{\mathbf{p}}$ ' problem; i.e., it is possible to find $k$ individual demand functions $\mathbf{x}^{1}(\mathbf{p}), \ldots, \mathbf{x}^{k}(\mathbf{p})$ such that

$$
\begin{aligned}
\mathbf{X}(\overline{\mathbf{p}})= & \mathbf{x}^{1}(\overline{\mathbf{p}})+\ldots+\mathbf{x}^{k}(\overline{\mathbf{p}}) \\
& \text { and } \\
D_{p} \mathbf{X}(\overline{\mathbf{p}})= & D_{p} \mathbf{x}^{1}(\overline{\mathbf{p}})+\ldots+D_{p} \mathbf{x}^{k}(\overline{\mathbf{p}})
\end{aligned}
$$

- if $n$ is odd, then for any $k \geq(n-1) / 2+1$, it is possible to find an economy with $k$ consumers that solves the 'at $\overline{\mathbf{p}}$ ' problem

Proof. Stems from Mantel's result and from Proposition 3.1, with $E=F \cap$ $X(p)^{\perp}$.

In other words, for any point $\overline{\mathbf{p}}$ and for any function $\mathbf{X}(p)$ with a negative Jacobian at $\overline{\mathbf{p}}$, only $n / 2+1$ agents (at most) are needed to construct an economy for which the aggregate demand takes, at $\overline{\mathbf{p}}$, the same value and the same first derivative as $X(p)$. The same result obtains in the excess demand case, with $n$ being replaced by $n-1$ since the argument must be applied in the hyperplane orthogonal to the price vector.

\section{An open question}

We now state the basic question:

Question: Let $\overline{\mathbf{p}}$ be any point in $\mathbb{R}_{+}^{n}$ and let $X(p)$ be some $C^{1}$ function satisfying adding-up and such that $D=D_{p} \mathbf{X}(\overline{\mathbf{p}})$ is negative semidefinite (so that it remains negative semidefinite within a neighbourhood 
of $\overline{\mathbf{p}}$ ). For any $k \geq n / 2+1$, is it possible to find an economy with $k$ consumers that solves the 'around $\overline{\mathbf{p}}$ ' problem; i.e., is it possible to find an open neighbourhood $\mathcal{V}(\overline{\mathbf{p}})$ of $\overline{\mathbf{p}}$ and $k$ individual demand functions $\mathbf{x}^{1}(\mathbf{p}), \ldots, \mathbf{x}^{k}(\mathbf{p})$ such that, for any $\mathbf{p}$ in $\mathcal{V}(\overline{\mathbf{p}})$

$$
\mathbf{X}(\mathbf{p})=\mathbf{x}^{1}(\mathbf{p})+\ldots+\mathbf{x}^{k}(\mathbf{p})
$$

Take some $\mathcal{V}(\overline{\mathbf{p}})$ in which $D_{p} \mathbf{X}(\overline{\mathbf{p}})$ remains negative semidefinite. From the previous result, we know that the 'at' problem has a solution at any point of $\mathcal{V}(\overline{\mathbf{p}})$ . The question is whether it is possible to select at each point a particular solution so as to generate a differentiable function over $\mathcal{V}(\overline{\mathbf{p}})$.

Note that the existing proof in Chiappori and Ekeland (1999) does not apply here. It can readily be checked that it requires $k \geq n$ even when the Jacobian of $X$ is negative definite. However, the result derived by Chiappori and Ekeland is stronger than what is needed here. Indeed, they show that the decomposition can be freely chosen at $\overline{\mathbf{p}}$, which is not necessary in our context.

Should the answer to the above question be yes, it would interestingly reduce the number of agents needed to generate any function as an aggregate demand. If, on the contrary, the answer is negative, then it should be possible to generate a counter example, i.e., to exhibit a function $X(p)$, the Jacobian of which is negative semidefinite, that cannot be locally decomposed as the aggregate market demand of an economy with $n / 2+1$ consumers, albeit it can be decomposed 'at' any point. 


\section{References}

\section{References}

[1] Andreu, J. (1983): "Rationalization of Market demand on Finite Domain", Journal of Economic Theory, 7

[2] Browning, M., and P.A. Chiappori (1998): "Efficient Intra-Household Allocations : a General Characterization and Empirical Tests", Econometrica, $66,1241-78$

[3] Chiappori, P.A., and I. Ekeland (1999) : "Aggregation and Market Demand : an Exterior Differential Calculus Viewpoint", Econometrica, forthcoming.

[4] Debreu, G. (1974): "Excess Demand Functions", Journal of Mathematical Economics, 1, 15-23

[5] Diewert, W.E. (1977), "Generalized Slutsky conditions for aggregate consumer demand functions", Journal of Economic Theory, 15, 353-62

[6] Geanakoplos, J., and H. Polemarchakis, (1980): "On the Disaggregation of Excess Demand Functions", Econometrica, 315-331

[7] Mantel, R. (1974): "On the Characterization of Aggregate Excess Demand", Journal of Economic Theory, 7, 348-53

[8] Mantel, R. (1976): "Homothetic preferences and community excess demand functions", Journal of Economic Theory 12, 1976, 197-201

[9] Mantel, R. : "Implications of economic theory for community excess demand functions", Cowles Foundation Discussion Paper n 451, Yale University, 1977

[10] Shafer, W. and H. Sonnenschein (1983), "Market Demand and Excess Demand Functions", chapter 14 in Kenneth Arrow and Michael Intriligator (eds), Handbook of Mathematical Economics, volume 2, Amsterdam: North Holland,1982, 670-93 
[11] Sonnenschein, H. (1973a): "Do Walras' Identity and Continuity Characterize the Class of Community Excess Demand Functions", Journal of Economic Theory, 345-54.

[12] Sonnenschein, H. (1973b): "The utility hypothesis and market demand theory", Western Economic Journal 11, 404-410

[13] Sonnenschein, H. (1974): "Market excess demand functions", Econometrica $40,549-563$ 


\section{A. Proof of proposition 3.1}

The argument is a direct generalization of that used in Browning and Chiappori (1998). First, consider the matrix $M=D-D^{\prime}$. It is antisymmetric, i.e., $M^{\prime}=$ $-M$. This has the following implications:

Lemma A.1. The eigenvalues of any antisymmetric matrix are either zero or complex. Moreover, $\mathbb{R}^{n}$ can be decomposed as the sum of $(S+1)$ orthogonal subspaces $E_{0}=\operatorname{ker} M, E_{1}, \ldots, E_{S}$ (where $S \leq n / 2$ ) such that each $E_{i}$ (for $i \geq 1$ ) is spanned by two eigenvectors corresponding to a pair of conjugate eigenvalues.

Proof. Let $\lambda$ be an eigenvalue of $M$ and $x$ a corresponding eigenvector, then $M x=\lambda x$ and $M \bar{x}=\bar{\lambda} \bar{x}$. Then $\bar{x}^{\prime} M=\left(M^{\prime} \bar{x}\right)^{\prime}=-(M \bar{x})^{\prime}=-\bar{\lambda} \bar{x}^{\prime}$. It follows that

$$
\bar{x}^{\prime} M x=\lambda \bar{x}^{\prime} x=-\bar{\lambda} \bar{x}^{\prime} x
$$

and either $\lambda=0$ or $\lambda=-\bar{\lambda}$ and $\lambda$ is complex. Complex eigenvalues come by conjugate pairs. If $w_{i}$ and $\bar{w}_{i}$ are two conjugate eigenvectors corresponding respectively to $\lambda_{i}$ and $\bar{\lambda}_{i}$, then $E_{i}$ is spanned by $w_{i}$ and $\bar{w}_{i}$ (or, equivalently, by $\left(w_{i}+\bar{w}_{i}\right)$ and $i\left(w_{i}-\bar{w}_{i}\right)$, which are both real). The decomposition result is standard, and obtains by induction. It is true for $n=1$. Also, assume it is true for all $n \leq N-1$, let us show that it holds for $N$ as well. It holds if $M$ is zero. If not, there exist some non zero eigenvalue $\lambda_{i}$, and one can define $E_{i}$ as above. Then define $F_{i}=E_{i}^{\perp} . \quad F_{i}$ is stable by $M$, for if $w^{\prime} e=0$ for all $e \in E_{i}$ then $(M w)^{\prime} e=w^{\prime} M^{\prime} e=-w^{\prime}(M e)=0$ since $M e \in E_{i}$. Since $\operatorname{dim} F_{i}=N-2, F_{i}$ can be decomposed. Finally, $\mathbb{R}^{n}=E_{i} \oplus F_{i}$, QED.

This leads to the following characterization of $M$ :

Lemma A.2. There exists $S$ vectors $u_{1}, \ldots, u_{S}$ and $S$ vectors $v_{1}, \ldots, v_{S}$, where $S \leq n / 2$, such that

$$
M=\sum_{i=1}^{S}\left(u_{i} v_{i}^{\prime}-v_{i} u_{i}^{\prime}\right)
$$

Proof. This stems from the previous Lemma and from the fact that, within each 2-dimensional subspace $E_{i}, M$ can be decomposed as $u_{i} v_{i}^{\prime}-v_{i} u_{i}^{\prime}$ (see Browning and Chiappori, 1998)

Note, in particular, that $2 S \leq n$, which implies that $S \leq n / 2$ if $n$ is even and $S \leq(n-1) / 2$ if $n$ is odd. 
Finally, define

$$
E=\left\{u_{1}\right\}^{\perp} \cap \ldots \cap\left\{u_{S}\right\}^{\perp}
$$

Note that $\operatorname{dim} E \geq n-S \geq n / 2$ if $n$ is even and $\operatorname{dim} E \geq k-S \geq(n+1) / 2$ if $n$ is odd. For any $u, v \in E$ we have that

$$
u^{\prime} M v=0 \Leftrightarrow u^{\prime} D v=u^{\prime} D^{\prime} v=v^{\prime} D u
$$

which means exactly that the restriction of $D$ to $E$ is symmetric. 